\title{
Genotoxicidad por exposición a cigarrillos en fumadores jóvenes en Colombia
}

\author{
Yexania Arboleda-Moreno, ${ }^{1,2}$ Luz Stella Hoyos, ${ }^{1}$ Silvio Carvajal ${ }^{1}$ \\ y Carlos H. Sierra-Torres ${ }^{2}$
}

Forma de citar Arboleda-Moreno Y, Hoyos LS, Carvajal S, Sierra-Torres CH. Genotoxicidad por exposición a cigarrillos en jóvenes fumadores en Colombia. Rev Panam Salud Publica. 2004;15(6):367-72.

RESUMEN Objetivo. Evaluar la frecuencia de aberraciones cromosómicas (AC) en los linfocitos de sangre periférica de jóvenes fumadores de cigarrillos de la ciudad de Popayán, Colombia.

Métodos. En este estudio citogenético de casos y testigos participaron 32 jóvenes fumadores de cigarrillos y 32 jóvenes no fumadores. Todos se encontraban entre los 19 y 29 años de edad y ninguno consumía drogas psicoactivas, padecía de enfermedades crónicas o infecciosas o había estado expuesto a quimioterapia, radioterapia o a agentes químicos en el ámbito ocupacional. Se aplicó una encuesta a fin de obtener información demográfica, ocupacional (tipo de empleo, tipo y tiempo de exposición a agentes químicos), estilo de vida (consumo de bebidas alcohólicas y de drogas psicoactivas) y hábitos tabáquicos (fumador actual o previo, número de cigarrillos que fumaba diariamente, tiempo que llevaba fumando y tipo de cigarrillos que fumaba). Se parearon los casos con los testigos según la edad ( \pm 5 años) y el sexo. El estudio microscópico de las AC a partir de cultivos de linfocitos se realizó bajo el microscopio óptico con un aumento de 100X. Se analizaron 100 células en metafase completa $(2 \mathrm{n}=46$ cromosomas $)$ por persona y se contaron las AC estructurales (quiebres cromatídicos y cromosómicos) y numéricas (cambio en el número de cromosomas). Se ajustó la frecuencia de AC en función del consumo de alcohol mediante un modelo lineal unifactorial.

Resultados. La frecuencia de AC totales fue significativamente mayor en los jóvenes fumadores de cigarrillos $(6,02 \pm 0,52)$ que en los que no fumaban $(3,04 \pm 0,50)$ y el mayor número de $A C(7,77 \pm 0,88)$ se encontró en los que fumaban más de 3,0 años-cajetilla. Además, se observó una relación entre dosis y efecto, demostrada por el aumento de la frecuencia de AC al aumentar la intensidad del consumo $\left(r^{2}=0,2257\right)$.

Conclusión. Se confirmó la presencia de una asociación entre el consumo de cigarrillos y las $A C$ en jóvenes que fumaban poco. Estos resultados se deben tomar en cuenta para formular las políticas nacionales de prevención del tabaquismo y para evaluar las consecuencias del hábito de fumar, tanto desde el punto de vista social, económico y ambiental, como desde el punto de vista de la salud de las generaciones futuras.

Palabras clave Tabaquismo, trastorno por uso de tabaco, aberraciones cromosómicas, Colombia.

1 Unidad de Toxicología Genética y Citogenética, Departamento de Biología, Facultad de Ciencias Naturales, Exactas y de la Educación, Universidad del Cauca, Popayán, Colombia.

2 Laboratorio de Genética Humana, Departamento de Ciencias Fisiológicas, Facultad de Ciencias de la Salud, Universidad del Cauca, Popayán, Colom- bia. La correspondencia debe dirigirse a Carlos $\mathrm{H}$. Sierra-Torres, Laboratorio de Genética Humana, Facultad de Ciencias de la Salud, Universidad del Cauca, Cra. 6 No. 14N-02, Popayán, Colombia. Telefax: (57-2) 820 9872. Correo electrónico: hsierra@ unicauca.edu.co
Se ha estimado que las muertes anuales asociadas con el consumo de cigarrillos en el mundo fueron de $3 \mathrm{mi}-$ llones en 1990 y de 4 millones en 1998, y se espera que alcancen la cifra de 8,4 
millones en 2020. Aproximadamente $70 \%$ de estas muertes ocurren en países en desarrollo (1). Aunque el tabaquismo se ha reducido en los países desarrollados, su prevalencia continúa aumentando en países de bajos y medianos ingresos $(2,3)$, especialmente en la población juvenil y en las mujeres (4). En Colombia, una encuesta realizada sobre el consumo de sustancias psicoactivas en jóvenes de 10 a 24 años de edad demostró que $35,9 \%$ de esta población había fumado cigarrillos alguna vez en la vida (5).

El humo de los cigarrillos es una mezcla compleja de sustancias que se encuentra en dos formas: la fase de partículas en forma de aerosol y la fase de vapor, que está compuesta de gases, como nitrógeno, oxígeno y monóxido de carbono (6). La fase de partículas está constituida por más de 4000 compuestos químicos diferentes, como el alquitrán y la nicotina. Entre estos compuestos se han identificado 60 sustancias que son cancerígenas tanto para los animales como para el ser humano. Algunas de ellas son los hidrocarburos aromáticos policíclicos, las N-nitrosaminas y las aminas aromáticas $(7,8)$.

Varios estudios realizados en fumadores activos demuestran que hay una asociación directa entre el consumo de cigarrillos y la mortalidad por cáncer de pulmón (9). Esos resultados también de muestran la relación entre la dosis (la intensidad y duración del consumo de cigarrillos) y el efecto (la aparición de diferentes tipos de cáncer de pulmón) (8). Además, los cigarrillos también causan otros problemas de salud, entre ellos la enfermedad pulmonar obstructiva crónica, enfermedades cardiovasculares, accidentes cerebrovasculares, úlcera péptica, diabetes, síntomas de abstinencia, infecciones respiratorias en niños, muerte súbita infantil y otros tipos de cáncer (10). Debido a este conjunto de problemas de salud y al hecho de que las tasas de prevalencia de tabaquismo se han incrementando, especialmente en los jóvenes, es importante evaluar los efectos genotóxicos de los cigarrillos, entre ellos las alteraciones cromosómicas que se observan en fumadores, para identificar posibles riesgos para la salud.
Los linfocitos de sangre periférica se emplean como células centinela ideales para identificar las aberraciones cromosómicas (AC) provocadas por agentes químicos, físicos o biológicos (11). La prueba de AC es una técnica citogenética que permite identificar cambios en la estructura normal o en el número de cromosomas (12). Esta técnica es muy útil para el seguimiento de poblaciones expuestas, ya que permite identificar sustancias químicas con propiedades mutagénicas y cancerígenas mediante la evaluación de la totalidad del genoma celular (13). Además, se ha demostrado que las AC están asociadas con diversas enfermedades, como el cáncer y algunas enfermedades genéticas (14). La prueba de AC en linfocitos humanos es de gran importancia porque permite evaluar las lesiones genéticas primarias que no se repararon y se acumularon durante varios años de exposición, las cuales se expresan después de la primera división celular in vitro (15).

El objetivo de esta investigación fue evaluar la frecuencia de AC en los linfocitos de sangre periférica de fumadores jóvenes de cigarrillos en la ciudad de Popayán, Colombia.

\section{MATERIALES Y MÉTODOS}

\section{Población objeto de estudio y toma de muestras}

Se realizó un estudio citogenético de casos y testigos en jóvenes fumadores de cigarrillos de la ciudad de Popayán, Colombia.

Se entrevistó a aproximadamente 500 personas mediante una breve encuesta y se preseleccionó a los jóvenes fumadores de cigarrillos y a los jóvenes no fumadores. A las personas seleccionadas se les aplicó una segunda encuesta para obtener información demográfica (edad, sexo y lugar de procedencia), antecedentes de salud e información ocupacional (estado de salud, tipo de empleo, tipo y tiempo de exposición a agentes químicos), estilo de vida (consumo de bebidas alcohólicas y de drogas psicoactivas) y hábitos tabáquicos (fumador actual o previo, número de cigarrillos que fumaba diariamente, tiempo que llevaba fumando y tipo de cigarrillos que fumaba). Los criterios de inclusión fueron tener entre 19 y 29 años de edad y no padecer de enfermedades crónicas o infecciosas. Se excluyó a las personas que consumían drogas psicoactivas, que padecían de enfermedades crónicas o infecciosas y que habían estado expuestas a quimioterapia, radioterapia o a agentes químicos en el ámbito ocupacional.

Después de obtener el consentimiento informado de los posibles participantes, se tomó una muestra de sangre de $20 \mathrm{~mL}$ por venopunción con jeringas heparinizadas, con el fin de establecer cultivos in vitro de linfocitos obtenidos de 32 casos y 32 testigos. Se parearon los casos y los testigos según la edad ( \pm 5 años) y el sexo. Se utilizó como indicador de exposición al tabaquismo la variable "años-cajetilla", definida como el número promedio de cajetillas de cigarrillos fumadas diariamente por una persona durante el número de años que mantuvo este hábito (16).

Todos los procedimientos de la investigación se realizaron teniendo en cuenta los principios bioéticos establecidos en la Declaración de Helsinki (17).

\section{Prueba de aberraciones cromosómicas}

Las muestras de sangre se utilizaron para establecer cultivos de linfocitos siguiendo procedimientos estándar en nuestro laboratorio (18). En resumen, a 4,5 mL de medio de cultivo RPMI-1640 completo (enriquecido con suero fetal bovino al $10 \%$, $2 \mathrm{mM}$ de L-glutamina, $100 \mathrm{U} / \mathrm{mL}$ de penicilina y $100 \mu \mathrm{g} / \mathrm{mL}$ de estreptomicina) se agregaron 0,5 $\mathrm{mL}$ de sangre y $0,2 \mathrm{~mL}$ de fitohemaglutinina, y la mezcla se incubó a $37^{\circ} \mathrm{C}$. A las 6 horas, a cada cultivo se le añadieron $50 \mu \mathrm{L}$ de 5,2-bromodeoxiuridina $(\mathrm{BrdU})(10 \mu \mathrm{M})$ para poder diferenciar las células en su primer, segundo y tercer ciclo de división celular. A las 50 horas, los cultivos se trataron con colquicina a una concentración final de $0,1 \mathrm{mg} / \mathrm{mL}$, a fin de bloquear la acción del huso mitótico y obtener 
un buen número de células en metafase. Finalmente, los cultivos se cosecharon a las 52 horas, se centrifugaron las células para eliminar el medio de cultivo (800-1 $000 \mathrm{rpm} / 7 \mathrm{~min})$, se agregó solución hipotónica $(0,075 \mathrm{M}$ de $\mathrm{KCl}$ ) a $37{ }^{\circ} \mathrm{C}$ durante 20 minutos para hinchar las células, y estas se fijaron con solución de Carnoy (metanol y ácido acético, 3:1). Las preparaciones citológicas se hicieron con 2-3 gotas de la suspensión celular concentrada en portaobjetos limpios y mantenidos en ácido acético frío al $60 \%$. Las placas se secaron cuidadosamente en una plancha de calor a $56^{\circ} \mathrm{C}$ durante $2 \mathrm{mi}-$ nutos y después de tres días se colorearon mediante el método de coloración diferencial con tinción de Giemsa (19). Posteriormente, las placas se montaron con Entellan, se codificaron y se sometieron a un doble enmascaramiento para evitar sesgos durante su evaluación. Para el análisis de las AC, las placas se observaron bajo el microscopio óptico a un aumento de 100X. Se analizaron 100 células en metafase completa ( $2 n=46$ cromosomas) por persona. Se contabilizaron las AC de tipo estructural (quiebres cromatídicos, cromosómicos o ambos) y numérico (cambio en el número de cromosomas) sin especificar el par o grupo de cromosomas en el que se detectó la aberración. Todos los reactivos utilizados fueron de alta pureza (Sigma Chemical, St. Louis, MO, EUA).

\section{Análisis estadístico}

Para el análisis estadístico se utilizó el programa SPSS para Windows, versión 10 (SPSS Inc., Chicago, IL, EUA). Las diferencias entre las medias de las variables de los casos y los testigos se evaluaron mediante la prueba de la $t$ de Student y la distribución de variables, mediante la prueba de ji al cuadrado. Se ajustó la frecuencia de AC en función del consumo de alcohol mediante un modelo lineal general unifactorial. La significación estadística se determinó mediante el análisis de la varianza y la corrección de Bonferroni para comparaciones múltiples cuando el valor de $F$ fue significativo (20). El análisis de regresión lineal permitió determinar la asociación entre el número de años-cajetilla fumados y el número de AC. Se utilizó un nivel de significación de 0,05.

\section{RESULTADOS}

\section{Características de la población de estudio}

Como se indica en el cuadro 1, 78\% de los fumadores eran hombres y $22 \%$, mujeres. La edad promedio de los fumadores fue de 22,84 $\pm 2,69$ años, con una mayor frecuencia entre los 22 y 25 años de edad (53\%). No se observaron diferencias significativas en las variables de pareamiento (sexo y edad) entre los casos y los testigos $(P>0,05)$. El número promedio de cigarrillos fumados diariamente fue de 8,53 $\pm 4,64$, con un tiempo promedio como fumador de 6,41 $\pm 2,30$ años. Todos los fumadores afirmaron que consumían bebidas alcohólicas, en comparación con solo $56 \%$ de los testigos. Por esta razón, se consideró el consumo del alcohol como variable de ajuste para los análisis estadísticos posteriores.

\section{Efecto genotóxico del consumo de cigarrillos}

La media del número de $\mathrm{AC}$ totales fue significativamente mayor en los fumadores de cigarrillos $(6,02 \pm 0,52)$ que en los jóvenes no fumadores $(3,04$ $\pm 0,50)$ y el mayor número de $\mathrm{AC}(7,77$ $\pm 0,88$ ) se encontró en los que fumaban más de 3,0 años-cajetilla (cuadro 2). Se observó que tanto en el grupo expuesto (fumadores) como en el grupo testigo (no fumadores), los quiebres cromatídicos (figura 1) eran el tipo de aberración más frecuente. De igual manera, se observó que el número de quiebres cromatídicos era significativamente mayor en los fumadores que en los testigos $(5,02 \pm 0,47$ frente a $2,63 \pm 0,47$, respectivamente). Este efecto dependió de la intensidad del consumo: 3,92 $\pm 0,77$ quiebres cromatídicos en los que fumaban de 0,3 a 1,5

CUADRO 1. Características de la población de fumadores y no fumadores estudiada. Popayán, Colombia

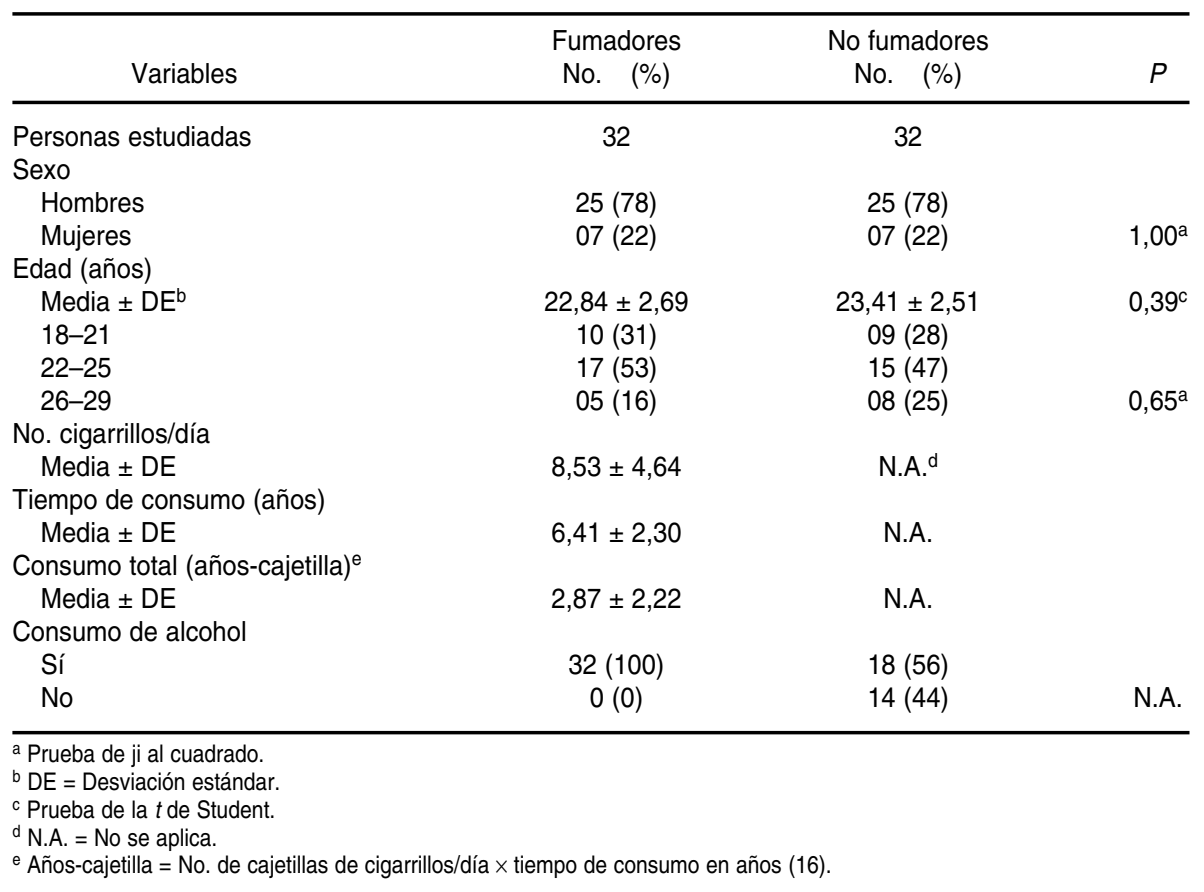


CUADRO 2. Número de aberraciones cromosómicas en no fumadores y fumadores, en función del número de añoscajetilla. Popayán, Colombia

\begin{tabular}{|c|c|c|c|c|c|c|}
\hline \multirow[b]{2}{*}{ Consumo } & \multicolumn{2}{|c|}{$\begin{array}{c}\text { Quiebres } \\
\text { cromatídicos }^{\mathrm{a}}\end{array}$} & \multicolumn{2}{|c|}{$\begin{array}{c}\text { Quiebres } \\
\text { cromosómicos }^{\mathrm{b}}\end{array}$} & \multicolumn{2}{|c|}{ Total de quiebres } \\
\hline & No. & $\begin{array}{l}\text { Media } \pm \text { error } \\
\text { estándarc }\end{array}$ & No. & $\begin{array}{l}\text { Media } \pm \text { error } \\
\text { estándarc }\end{array}$ & No. & $\begin{array}{c}\text { Media } \pm \text { error } \\
\text { estándarc }\end{array}$ \\
\hline $\begin{array}{l}\text { No fumador } \\
\text { Fumador }\end{array}$ & 32 & $2,63 \pm 0,47$ & 11 & $1,13 \pm 0,27$ & 32 & $3,04 \pm 0,50$ \\
\hline (años-cajetilla) & 31 & $5,02 \pm 0,47^{d}$ & 23 & $1,63 \pm 0,18$ & 32 & $6,02 \pm 0,52^{d}$ \\
\hline $0,3-1,5$ & 10 & $3,92 \pm 0,77$ & 70 & $1,70 \pm 0,32$ & 11 & $4,62 \pm 0,80$ \\
\hline $1,6-3,0$ & 12 & $5,02 \pm 0,71^{e}$ & 80 & $1,48 \pm 0,30$ & 12 & $5,98 \pm 0,77^{e}$ \\
\hline$P \quad>3,0$ & 9 & $\begin{array}{c}6,24 \pm 0,81^{e} \\
0,001\end{array}$ & 80 & $\begin{array}{c}1,73 \pm 0,30 \\
0,418\end{array}$ & 9 & $\begin{array}{c}7,77 \pm 0,88^{e} \\
0,001\end{array}$ \\
\hline
\end{tabular}

años-cajetilla; $5,02 \pm 0,71$, en los que fumaban entre 1,6 y 3,0 años-cajetilla; y $6,24 \pm 0,81$, en los que fumaban más de 3,0 años-cajetilla (cuadro 2). Respecto al número de quiebres cromosómicos, no se encontraron diferencias estadísticamente significativas entre los jóvenes fumadores $(1,63 \pm 0,18) \mathrm{y}$ los no fumadores $(1,13 \pm 0,27)$. Es importante aclarar que en las personas estudiadas no se detectaron aberraciones de tipo numérico (por ejemplo, poliploidias) ni otros tipos de aberraciones estructurales (tales como figuras trirradiales o tetrarradiales).

FIGURA 1. Metafase con quiebre cromatídico

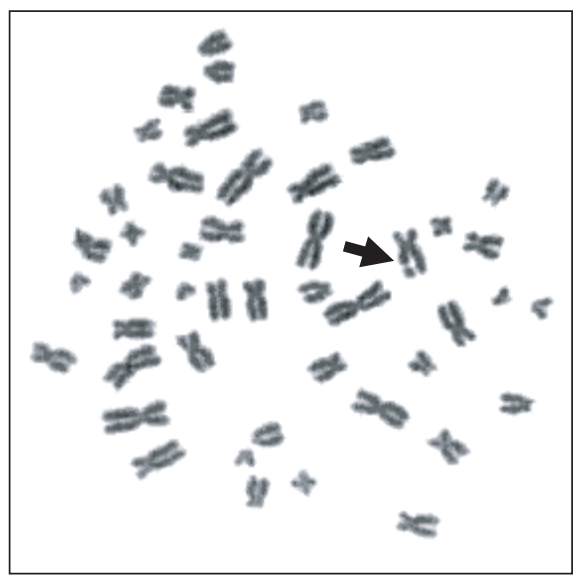

Se observó una relación entra la dosis y el efecto (figura 2), manifestada en el incremento de la frecuencia de AC a medida que aumentaba el consumo de cigarrillos en años-cajetilla (incluidos los jóvenes que no fumaban). El coeficiente de determinación $\left(r^{2}=0,2257\right)$ permite inferir que la variabilidad observada en el número total de AC se debe en $22,57 \%$ de los casos a la variabilidad en el número de años-cajetilla fumados.

\section{DISCUSIÓN}

El consumo de cigarrillos es una de las principales causas de enfermedad y muerte en todo el mundo (1). La diversidad y las características de los compuestos que contienen los cigarrillos han sido objeto de diversas investigaciones relacionadas con los mecanismos patógenos del tabaquismo (6). En el presente estudio, la mayor proporción de fumadores fueron hombres entre las edades de 22 y 25 años. La alta prevalencia del consumo de cigarrillos en los jóvenes colombianos (5) y el incremento del número de casos nuevos de cáncer de pulmón (21) justifican la necesidad de evaluar el potencial genotóxico de los cigarrillos en nuestra población.

La mayor frecuencia de AC encontradas en este estudio en fumadores en comparación con los no fumadores, así como la asociación observada entre el consumo de cigarrillos y la frecuencia de alteraciones del material genético (ADN), ponen de manifiesto el riesgo que representa el tabaquismo para la salud de la población, en particular para los jóvenes. Estas observaciones se suman a las de otros estudios que demuestran la actividad mutagénica de los compuestos químicos que contienen los cigarrillos (22) y la asociación del tabaco con varias enfermedades (10). Además, estudios prospectivos de cohorte realizados en Italia y el norte de Europa indican que existe una asociación estadísticamente significativa entre las AC y el riesgo de padecer de cáncer (14).

Esta investigación confirma los resultados de otros estudios realizados en fumadores de cigarrillos. En Estados Unidos, varios autores encontraron una frecuencia de AC significativamente mayor en fumadores con cáncer de pulmón en comparación con fumadores sin cáncer y con no fumadores (11, 23-25). En China, un estudio demostró que la frecuencia de AC y de micronúcleos era significativamente mayor en fumadores que en no fumadores (26). En una investigación anterior realizada en nuestro laboratorio (27) en la que se estudió a personas mayores de 30 años de edad se encontró que los fumadores $(19,25 \pm 15,56$ 
FIGURA 2. Análisis de correlación entre el número de aberraciones cromosómicas y el número de años-cajetilla. Popayán, Colombia

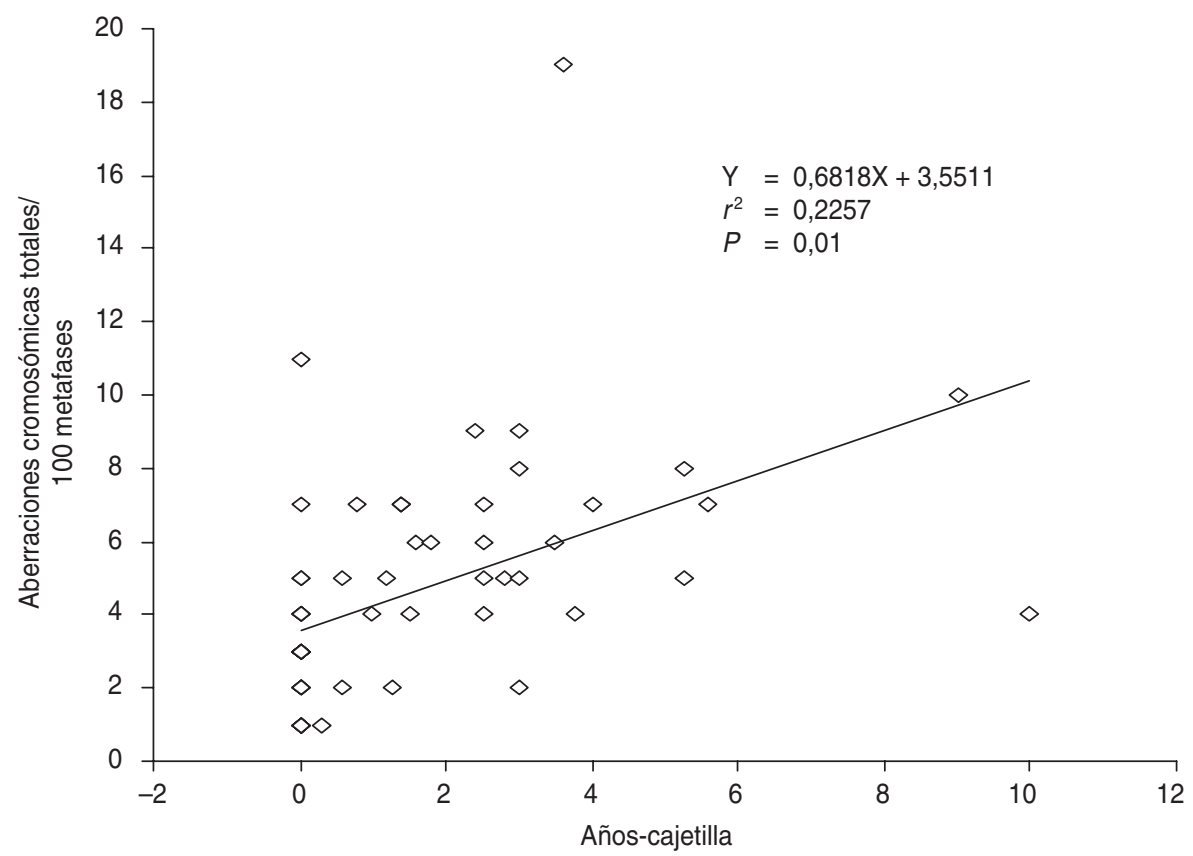

años-cajetilla) presentaban una mayor frecuencia de $\mathrm{AC}$ que los no fumadores $(12,2 \pm 3,33$ frente a $3,44 \pm 2,60$, respectivamente; $P<0,01)$. Además de confirmar la asociación entre el consumo de cigarrillos y el incremento en la frecuencia de AC previamente observada, el presente estudio demuestra que el consumo "leve" de cigarrillos $(2,87 \pm$ 2,22 años-cajetilla) también puede producir daño cromosómico.

El tipo de AC que puede producir un agente químico depende básica- mente de la etapa del ciclo celular en la que se encuentran los linfocitos durante la exposición (15). En este estudio se encontró una mayor frecuencia de quiebres cromatídicos en comparación con quiebres cromosómicos (cuadro 2). Esta elevada frecuencia de quiebres cromatídicos en la población de fumadores de cigarrillos podría ser causada por el efecto de los residuos de sustancias genotóxicas acumuladas en las células. Este efecto dañino se expresa en forma de $\mathrm{AC}$, que se produ- cen cuando las células pasan por la fase de síntesis en su ciclo celular (15).

En los estudios de monitoreo de poblaciones expuestas, el empleo de marcadores biológicos, como las AC, puede ayudar a fortalecer las estrategias de prevención, al permitir identificar poblaciones en riesgo de padecer diferentes enfermedades (28). Para implementar estas estrategias también es necesario contar con leyes, basadas en datos científicos obtenidos localmente, que fomenten la vigilancia y el control de los problemas de salud (29).

En este contexto, este es el primer estudio publicado que demuestra el efecto genotóxico del consumo de cigarrillos en una población de jóvenes colombianos que fuman relativamente poco. Estos resultados deben tomarse en cuenta para formular las políticas nacionales de prevención del tabaquismo y para evaluar sus consecuencias, tanto desde el punto de vista social, económico y ambiental, como desde el punto de vista de la salud de las generaciones futuras.

Agradecimientos: Los autores expresan su agradecimiento a las vicerrectorías de Investigaciones y Administrativa, a la Fundación de Apoyo y al Museo de Historia Natural de la Universidad del Cauca por su apoyo económico. También agradecen al Departamento de Biología y a la Unidad de Toxicología Genética y Citogenética de la Universidad del Cauca por su colaboración y asistencia en el desarrollo de esta investigación.

\section{REFERENCIAS}

1. World Health Organization. The world health report 2002: reducing risks, extending healthy life. Geneva: WHO; 2002.

2. Corrao MA, Guindon GE, Cokkinides V, Sharma N. Building the evidence base for global tobacco control. Bull World Health Organ. 2000;78:884-90.

3. Ramsay S. PAHO exposes tobacco-industry tactics in Latin America. Lancet. 2002;360: 2057.

4. Da Costa e Silva VL, Koifman S. Smoking in Latin America: a major public health problem. Cad Saude Publica. 1998;14:99-108.
5. RUMBOS: Programa presidencial para afrontar el consumo de drogas. Sondeo nacional del consumo de drogas en jóvenes. Santa Fe de Bogotá: Presidencia de la República de Colombia; 1999.

6. Baker RR. Product formation mechanisms inside a burning cigarette. Progr Energy Combustion Sci. 1981;7:135-53.

7. International Agency for Research on Cancer. Monograph on the evaluation of the carcinogenic risk of chemicals to humans. Lyon: IARC; 1986
8. Hoffmann D, Hoffmann I. The changing cigarette, 1950-1995. J Toxicol Environ Health. 1997;50:307-64.

9. Travis WD, Travis LB, Devesa SS. Lung cancer. Cancer. 1995;75:191-202.

10. Fagerstrom K. The epidemiology of smoking: health consequences and benefits of cessation. Drugs. 2002;62:1-9.

11. Au WW, Walker DM, Ward JBJ, Whorton E, Legator MS, Singh V. Factors contributing to chromosome damage in lymphocytes of cigarette smokers. Mutat Res. 1991;260:137-44. 
12. Natarajan AT, Obe G. Screening of human populations for mutations induced by environmental pollutants: use of human lymphocyte system. Ecotoxicol Environ Saf. 1980;4: 468-81.

13. Au WW, Cajas-Salazar N, Salama S. Factors contributing to discrepancies in population monitoring studies. Mutat Res. 1998;400: 467-78.

14. Bonassi S, Hagmar L, Stromberg U, Montagud $\mathrm{AH}$, Tinnerberg H, Forni A, et al. Chromosomal aberrations in lymphocytes predict human cancer independently of exposure to carcinogens. European Study Group on Cytogenetic Biomarkers and Health. Cancer Res. 2000;60:1619-25.

15. Evans HJ, Scott D. Influence of DNA synthesis on the production of chromatid aberrations by $X$ rays and maleic hydrazide in Vicia faba. Genetics. 1964;49:17-38.

16. Sierra-Torres $\mathrm{CH}, \mathrm{Au} \mathrm{WW}$, Arrastia CD, Cajas-Salazar N, Robazetti SC, Payne DA, et al. Polymorphisms for chemical metabolizing genes and risk for cervical neoplasia. Environ Mol Mutagen. 2003;41:69-76.

17. The Helsinki Declaration. Orv Hetil. 1965;106: 1715-16.

18. Hoyos LS, Carvajal S, Solano L, Rodriguez J, Orozco L, Lopez Y, et al. Cytogenetic monitoring of farmers exposed to pesticides in Colombia. Environ Health Perspect. 1996;104: 535-8.
19. Goto K, Akematsu T, Shimazu H, Sugiyama T. Simple differential Giemsa staining of sister chromatids after treatment with photosensitive dyes and exposure to light and the mechanism of staining. Chromosoma. 1975;53:223-30.

20. Whorton EBJ. Some experimental design and analysis considerations for cytogenetics studies. Environ Mutagen. 1985;7(Suppl 4):9-15.

21. Colombia, Ministerio de Salud. Segundo estudio nacional de factores de riesgo de enfermedades crónicas. Santa Fe de Bogotá: Centro Nacional de Consultoría; 1999.

22. Sorsa M. Experimental studies on the mutagenicity and related effects of low-tar and high-tar cigarettes in relation to smoker exposures. IARC Sci Publ. 1986;38:227-35.

23. Conforti-Froes N, el-Zein R, Abdel-Rahman SZ, Zwischenberger JB, Au WW. Predisposing genes and increased chromosome aberrations in lung cancer cigarette smokers. Mutat Res. 1997;379:53-9.

24. El-Zein R, Zwischenberger JB, Wood TG, Abdel-Rahman SZ, Brekelbaum C, Au WW. Combined genetic polymorphism and risk for development of lung cancer. Mutat Res. 1997; 381:189-200.

25. Cajas-Salazar N, Au WW, Zwischenberger JB, Sierra-Torres CH, Salama SA, Alpard SK, et al. Effect of epoxide hydrolase polymorphisms on chromosome aberrations and risk for lung cancer. Cancer Genet Cytogenet. 2003;145:97-102.
26. Jin $\mathrm{YL}$, Wang HZ, Gu H. Observation of chromosome aberration and micronucleus formation in peripheral blood lymphocytes among cigarette smokers. Zhonghua Liu Xing Bing Xue Za Zhi. 1997;18:40-2.

27. Sierra-Torres MS. Alteraciones en la frecuencia de aberraciones cromosómicas en linfocitos de fumadores de cigarrillos. Trabajo de grado de Biología. Departamento de Biología, Facultad de Ciencias Naturales, Exactas y de la Educación, Universidad del Cauca; 1999.

28. Au WW, Badary OA, Heo MY. Cytogenetic assays for monitoring populations exposed to environmental mutagens. Occup Med. 2001; 16:345-57.

29. Shibuya K, Ciecierski C, Guindon E, Bettcher DW, Evans DB, Murray CJ. WHO Framework Convention on Tobacco Control: development of an evidence based global public health treaty. Br Med J. 2003;327:154-7.

Manuscrito recibido el 4 de noviembre de 2003. Aceptado para publicación, tras revisión, el 28 de abril de 2004.

ABSTRACT Objective. To evaluate the frequency of chromosome aberrations (CAs) in the peripheral blood lymphocytes of young cigarette smokers in the city of Popayán, Colombia.

\section{Genotoxicity from exposure to cigarettes in young smokers in Colombia}

Methods.

In this cytogenetic case-control study there were 32 young cigarette smokers and 32 nonsmokers. All of them were between 19 and 29 years old and none used psychoactive drugs, suffered from chronic or infectious diseases, or had been exposed to chemotherapy or radiation therapy or to chemical agents in their work. A survey was used to obtain demographic information, occupational information (type of employment, type of and length of exposure to chemical agents), lifestyle information (consumption of alcoholic beverages and psychoactive drugs), and information on smoking (current or former smoker, number of cigarettes smoked daily, length of time smoking, and type of cigarettes smoked). The cases were matched with the controls by age ( \pm 5 years) and sex. The microscopic study of the CAs using lymphocyte cultures was carried out under the light microscope with 100X magnification. For each study participant, 100 complete metaphase cells ( $2 n=46$ chromosomes) were analyzed, counting the structural CAs (chromatid breaks and chromosome breaks) and numerical CAs (change in the number of chromosomes). The frequency of CAs was adjusted for alcohol consumption, using a univariate linear model.

Results. The frequency of total CAs was significantly greater in the young cigarettes smokers $(6.02 \pm 0.52)$ than in the nonsmokers $(3.04 \pm 0.50)$, and the greatest number of CAs $(7.77 \pm 0.88)$ was found in those who had a pack-year value of more than 3.0. In addition, there was a dose-effect relationship, shown by the increase in the frequency of CAs with an increase in the pack-years of consumption (coefficient of determination $=0.2257)$.

Conclusions. We confirmed the association between cigarette consumption and CAs in young people who smoked relatively little. These results should be taken into account in order to formulate national smoking prevention policies and to evaluate their outcomes, from both the social, economic, and environmental standpoint and the standpoint of the health of future generations. 